\title{
Genetic variation of morphological traits in Drosophila melanogaster under poor nutrition: isofemale lines and offspring-parent regression
}

\author{
OLEG A. BUBLIY $\uparrow$, VOLKER LOESCHCKE* + \& ALEXANDRA G. IMASHEVA $\dagger$ \\ $\dagger$ Vavilov Institute of General Genetics, Gubkin Street 3, 117809 Moscow, Russia and $\ddagger$ Department of Ecology \\ and Genetics, University of Aarhus, Ny Munkegade, Building 540, DK-8000 Aarhus C, Denmark
}

\begin{abstract}
Variation of three morphological traits (thorax length, wing length and sternopleural bristle number) was examined in Drosophila melanogaster reared on a medium with low yeast content and on a standard medium using the isofemale line analysis and offspring-parent regression. The aim was to test whether these experimental approaches give different patterns of changes in genetic variability estimates when stressful and nonstressful environments are compared. Heritabilities and genetic and phenotypic variances were generally higher in the isofemale line design than in the offspring-parent regression design under both standard and poor nutritional conditions. For each trait, the response of heritability to stress was similar in both designs: wing length exhibited lower heritability under poor nutrition, whereas heritabilities of thorax length and sternopleural bristle number did not differ between nutritional regimes. Statistically significant differences in the genetic variances and the environmental variances between stressful and nonstressful environments were recorded only in isofemale lines: the genetic variance of thorax length and the environmental variances of thorax length and wing length were higher under poor nutrition. The results are compared to literature data and possible reasons of increased genetic variability estimates in isofemale lines are briefly discussed.
\end{abstract}

Keywords: Drosophila melanogaster, genetic variation, heritability, morphological traits, nutritional stress.

\section{Introduction}

The level of genetic differentiation of populations caused by selection is determined by the amount of additive genetic variation. Extensive empirical evidence indicates that the proportion of additive variation in the total phenotypic variation, i.e. the narrow-sense heritability, may change with changing environments, thus allowing for an acceleration or deceleration of the evolutionary rate (Hoffmann \& Parsons, 1991). Several hypotheses have been advanced to explain an increase or a decrease of additive genetic variation/heritability under adverse conditions (see Hoffmann \& Parsons, 1991; Hoffmann \& Merilä, 1999, for reviews). The most popular among these is the so-called 'selection history hypothesis', which suggests an increase in heritability under adverse conditions (Mather, 1943; Holloway et al., 1990;

\footnotetext{
*Correspondence. E-mail: volker@biology.au.dk
}

Pigliucci et al., 1995). According to this hypothesis, higher heritability in a novel (and presumably stressful) environment is due to the expression of the additive variation not eliminated by stabilizing selection because in the past the population was rarely, if ever, subjected to this environment. Alternatively, a decrease in heritability under stress is usually explained by a drastic increase in environmental variance, which is often the case when stress promotes individual differences in growth rate (Blum, 1988).

Recent studies of genetic variation of morphological traits in Drosophila reported both higher and lower genetic variances/heritabilities in extreme environments. The results of some of these studies were conflicting: the same traits exposed to similar stressors often exhibited opposite trends in different experiments. For instance, De Moed et al. (1997), who estimated genetic variation in D. melanogaster using the isofemale line technique (see Hoffmann \& Parsons, 1988), showed that a 
combined stress (poor nutrition and low temperature) enhanced the genetic component of variation in thorax length and wing length. Hoffmann \& Schiffer (1998) and Woods et al. (1999) obtained different results using offspring-parent comparisons in an outbred population subjected to a combined stress with a nutritional component (poor nutrition, ethanol added to the medium and repeated cold shock). In these experiments, genetic variance of wing traits and bristles did not change, whereas the heritabilities either remained constant or decreased. A similar pattern of genetic variability estimates in wing length and sternopleural bristle number (but not thorax length) were recorded by Bubliy et al. (2000b), who analysed genetic variation under poor nutritional conditions using the half-sib design. In another study of nutritional stress in isofemale lines, Imasheva et al. (1999) showed that genetic variance of most morphological traits examined (thorax length, wing length and three meristic traits) increased under food-limited conditions and the heritabilities did not change or decreased.

Investigations of the effect of temperature stress on genetic variation in Drosophila also produced controversial results. Isofemale line studies (Barker \& Krebs, 1995; Imasheva et al., 1998; Loeschcke et al., 1999) demonstrated a trend towards higher heritabilities of thorax length, wing length and sternopleural bristle number in flies reared at extreme temperatures. By contrast, when genetic variation was assessed by offspringparent regression (wing length; Sgrò \& Hoffmann, 1998a) or artificial directional selection (sternopleural bristles; Bubliy et al., 2000a), heritabilities were not affected by stress.

Based on the above examples one can suggest that the isofemale line analysis typically reveals a trend for higher genetic variation under stress, whereas other quantitative genetics techniques do not detect any change, or show a change in the opposite direction. To test this assumption, here we compared estimates of genetic variation obtained for the same population of D. melanogaster by isofemale line analysis and offspring-parent regression under stressful and nonstressful conditions. Variation in three morphological traits, thorax length, wing length and sternopleural bristle number, was examined. As a stress factor, we used poor nutrition due to a low percentage of yeast in the medium. In order to monitor the magnitude of stress to which populations were subjected, three parameters, larva-to-adult viability, adult body size and phenotypic variance, were considered. Viability and body size are associated with fitness and thus measure stress levels directly since stress by definition is related to a reduction in fitness (Forbes \& Calow, 1997). Phenotypic variation (variance or coefficient of variation) often increases as environmental conditions deteriorate and may be an indicator of stress at the population level (see Møller \& Swaddle, 1997; Woods et al., 1999).

\section{Materials and methods}

Flies were collected on a fruit bait in a wild D. melanogaster population inhabiting a small Moscow orchard near the Vavilov Institute of General Genetics in late August 1999. Immediately after collection, 50 females were used to found isofemale lines, and 200 pairs were placed in 20 bottles to set up an outbred population, which will be hereafter referred to as the 'mixed population'. Before the experiments, the isofemale lines and the mixed population were maintained for three generations on a standard sugar-yeast-agar medium in glass bottles $(30 \mathrm{~mL}$ of medium $)$ at $25^{\circ} \mathrm{C}$. In each isofemale line, each subsequent generation was founded by 30 pairs of nonvirgin flies aged 5-7 days that were placed in a bottle for several hours to lay eggs. In the mixed population, virgin flies were collected each generation, aged for 5-7 days, then mixed and distributed among 20 bottles ( 20 flies per bottle), where they were left for several hours to lay eggs. Standard Drosophila medium, subsequently used as a nonstressful environment, contained $32 \mathrm{~g}$ of dry yeast, $54 \mathrm{~g}$ of sugar and $16 \mathrm{~g}$ of agar per $1 \mathrm{~L}$ of water; $1 \mathrm{~mL}$ of acetic acid, $10 \mathrm{~mL}$ of Nipagin and $10 \mathrm{~mL}$ of penicillin were added against bacterial and fungal infection. As a stressful environment hereafter referred to as poor, we used a medium of the same content except for the yeast concentration ( $2 \mathrm{~g}$ of dry yeast per $1 \mathrm{~L}$ of water).

To obtain flies for estimating genetic variation, we placed in empty vials plastic spoons each filled with $2 \mathrm{~mL}$ of standard medium supplemented with live yeast. In each vial, the appropriate number (see below) of flies aged 5-8 days were placed for $24 \mathrm{~h}$ to lay eggs. Then, the spoons were removed and first-instar larvae transferred to vials (20 larvae per vial) with $7 \mathrm{~mL}$ of medium (standard or poor, according to the experimental design) without live yeast. On the poor medium, the development time was significantly longer (on average 15 days vs. 9.5 days on the standard medium).

In the isofemale line experiment, 30 pairs of virgin flies were mated to serve as parents for each line. The larvae obtained for each line were transferred to four vials, two with the standard and two with the poor medium. In the offspring-parent regression experiment, 1000 pairs of virgin flies were collected in the mixed population. These flies were pooled and randomly assigned to 100 groups with 10 flies in each group. Larvae obtained from each of the groups were transferred to a vial with the standard and a vial with the poor medium. The emerged adults were sexed, males 
and females separated to ensure virginity, and pooled within each treatment. Within each treatment, 200 pairs were randomly formed. The larvae obtained from each pair were transferred to a vial with standard or poor medium depending on the environments experienced by the parents.

All flies collected in both experiments were stored in a fixative liquid ( $1 / 2$ glycerine $+1 / 270 \%$ ethanol) for subsequent measurement of three morphological traits: thorax length, wing length and sternopleural bristle number. Thorax length (from the anterior margin of the thorax to the posterior tip of the scutellum from the dorsal view) and wing length (length of the longitudinal vein L3 from the intersection with the anterior crossvein to the tip of the wing) were measured in arbitrary micrometer units (70 units $=1 \mathrm{~mm})$ using an ocular micrometer and converted into $\mathrm{mm} \times 100$. Bilateral traits (wing length and bristle number) were measured on the left side of the fly. In the isofemale line experiment, three flies per vial (only females) were measured; in the offspring-parent regression experiment, we analysed 150 families (both parents and one male and one female offspring). The statistical treatment was conducted on untransformed data using procedures described by Zar (1996).

\section{Results}

\section{Viability and phenotypic variation}

Table 1 shows the effect of nutritional stress on viability in the isofemale lines and the parental and $F_{1}$ generation of the offspring-parent regression experiment. As seen from the table, mean viability per vial was lower under poor nutrition both in the isofemale lines and in the mixed population. The three viability estimates under poor nutrition were also significantly different (twotailed Mann-Whitney $U$-tests, $P<0.001$ ): viability was lowest in isofemale lines and highest in the parental generation of the mixed population. Under standard nutrition only viability differences between the isofemale lines and the offspring generation were statistically significant (two-tailed Mann-Whitney $U$-test, $Z=3.48$, $P<0.001)$.
Estimates of phenotypic variation of the three morphological traits in the poor and standard nutritional regimes are shown in Table 2 . In all cases, mean trait values were significantly lower under stressful conditions. In both regimes, means in isofemale lines typically were different from those in the mixed population (nine comparisons out of 12 , two-tailed $t$-tests, $P<0.001$ ), thorax length and bristle number values were greater in the mixed population, and wing length in isofemale lines. There were also differences between the two generations of the mixed population: flies from the offspring generation had smaller thorax length and wing length under poor nutrition $(t=11.27$, d.f. $=298$, $P<0.001$ and $t=8.28$, d.f. $=298, P<0.001$, respectively) and smaller thorax length under standard nutrition $(t=3.05$, d.f. $=298, P=0.002)$.

Differences in phenotypic variances between environments (Table 2) were not as clear as in mean values. When the flies were reared under poor nutrition, the phenotypic variances were higher in thorax length in the isofemale lines and the offspring generation of the mixed population, as well as in wing length in both generations of the mixed population. The bristle number in the offspring generation showed an opposite trend, but when the coefficient of variation $(\mathrm{CV})$ was used instead of the variance, the difference in phenotypic variation between nutritional regimes was nonsignificant ( $Z$-test, $Z=1.45, P=0.146$ ). In other cases, the use of $\mathrm{CV}$ did not change the direction or significance of differences between regimes. Variances in isofemale lines significantly (two-tailed $F$-tests, $P<0.001$ ) differed from variances in the mixed population in seven out of 12 comparisons; in the isofemale lines, variances were always higher. Differences between the two generations of the mixed population were observed only in thorax length under standard nutrition: flies from the offspring generation exhibited higher variability $\left(F_{149,149}=2.09\right.$, $P<0.001)$.

\section{Genetic variation in isofemale lines}

Table 3 presents the results of the two-way nested ANOVA for 50 isofemale lines in each of the nutritional regimes. A statistically significant among-line effect,

Table 1 Mean viabilities per vial $( \pm \mathrm{SE})$ in Drosophila melanogaster from isofemale lines and a mixed population (offspring-parent regression design)

\begin{tabular}{lccc}
\hline Experimental population and no. of vials per treatment & Standard nutrition & Poor nutrition & Comparison, $P \dagger$ \\
\hline Isofemale lines $(N=100)$ & $0.895 \pm 0.013$ & $0.634 \pm 0.017$ & $<0.001$ \\
Mixed population: parents $(N=100)$ & $0.930 \pm 0.017$ & $0.849 \pm 0.014$ & $<0.001$ \\
Mixed population: offspring $(N=150)$ & $0.938 \pm 0.009$ & $0.717 \pm 0.009$ & $<0.001$ \\
\hline
\end{tabular}

$\dagger$ Two-tailed probability, Mann-Whitney $U$-test.

(c) The Genetics Society of Great Britain, Heredity, 86, 363-369. 
Table 2 Means and phenotypic variances $\left(s^{2}\right)$ for three morphological traits in females of Drosophila melanogaster from isofemale lines and mixed population (offspring-parent regression design)

\begin{tabular}{|c|c|c|c|c|c|c|c|}
\hline \multirow{2}{*}{$\begin{array}{l}\text { Exp. population and } \\
\text { no. of flies per treatment }\end{array}$} & \multirow[b]{2}{*}{ Trait } & \multicolumn{2}{|c|}{ Standard nutrition } & \multicolumn{2}{|c|}{ Poor nutrition } & \multicolumn{2}{|c|}{ Comparison, $P \dagger$} \\
\hline & & Mean \pm SE & $s^{2}$ & Mean \pm SE & $s^{2}$ & Mean & $s^{2}$ \\
\hline \multirow{3}{*}{$\begin{array}{l}\text { Isofemale lines } \\
\qquad(N=300)\end{array}$} & Thorax length & $98.11 \pm 0.21$ & 12.72 & $81.99 \pm 0.33$ & 33.55 & $<0.001$ & $<0.001$ \\
\hline & Wing length & $136.58 \pm 0.58$ & 101.11 & $117.90 \pm 0.54$ & 88.94 & $<0.001$ & 0.268 \\
\hline & Bristle number & $10.07 \pm 0.07$ & 1.65 & $8.91 \pm 0.07$ & 1.51 & $<0.001$ & 0.420 \\
\hline \multirow{3}{*}{$\begin{array}{l}\text { Mixed population: } \\
\text { parents }(N=150)\end{array}$} & Thorax length & $102.09 \pm 0.33$ & 16.01 & $87.11 \pm 0.32$ & 15.55 & $<0.001$ & 0.858 \\
\hline & Wing length & $132.83 \pm 0.27$ & 10.68 & $119.19 \pm 0.32$ & 15.88 & $<0.001$ & 0.016 \\
\hline & Bristle number & $10.57 \pm 0.10$ & 1.41 & $9.55 \pm 0.09$ & 1.16 & $<0.001$ & 0.232 \\
\hline \multirow{3}{*}{$\begin{array}{l}\text { Mixed population: } \\
\text { offspring }(N=150)\end{array}$} & Thorax length & $100.88 \pm 0.23$ & 7.68 & $82.16 \pm 0.30$ & 13.34 & $<0.001$ & $<0.001$ \\
\hline & Wing length & $132.35 \pm 0.26$ & 10.15 & $115.06 \pm 0.38$ & 21.37 & $<0.001$ & $<0.001$ \\
\hline & Bristle number & $10.39 \pm 0.10$ & 1.55 & $9.31 \pm 0.08$ & 0.98 & $<0.001$ & 0.005 \\
\hline
\end{tabular}

$\dagger$ Two-tailed probability; comparisons were carried out by $t$-test (means) and $F$-test (variances).

Table 3 Mean squares and variance components $\left(s^{2}\right)$ from two-way nested ANova of three morphological traits for 50 isofemale lines of Drosophila melanogaster

\begin{tabular}{|c|c|c|c|c|c|c|c|}
\hline \multirow[b]{2}{*}{ Trait } & \multirow{2}{*}{$\begin{array}{l}\text { Source of } \\
\text { variation }\end{array}$} & \multirow[b]{2}{*}{ d.f. } & \multicolumn{2}{|c|}{ Standard nutrition } & \multicolumn{2}{|c|}{ Poor nutrition } & \multirow{2}{*}{$\begin{array}{l}\text { Comparison } \\
\text { of } s^{2}, P\end{array}$} \\
\hline & & & MS & $s^{2}$ & MS & $s^{2}$ & \\
\hline \multirow[t]{3}{*}{ Thorax length } & Among lines & 49 & 18.54 & 0.76 & $91.11^{* *}$ & 7.85 & $<0.001$ \\
\hline & Among vials & 50 & 13.98 & 1.00 & $44.04 * * *$ & 9.07 & $<0.001$ \\
\hline & Within vials & 200 & 10.98 & 10.98 & 16.82 & 16.82 & 0.001 \\
\hline \multirow[t]{3}{*}{ Wing length } & Among lines & 49 & $540.37^{* * *}$ & 86.98 & $387.16^{* * *}$ & 52.78 & 0.084 \\
\hline & Among vials & 50 & 18.46 & 1.44 & $70.51^{* * *}$ & 16.67 & $<0.001$ \\
\hline & Within vials & 200 & 14.15 & 14.15 & 20.49 & 20.49 & 0.004 \\
\hline \multirow[t]{3}{*}{ Bristle number } & Among lines & 49 & $3.03 * * *$ & 0.33 & $3.31 * * *$ & 0.39 & 0.610 \\
\hline & Among vials & 50 & 1.02 & -0.15 & 0.98 & -0.07 & - \\
\hline & Within vials & 200 & 1.48 & 1.48 & 1.20 & 1.20 & 0.072 \\
\hline
\end{tabular}

$* P<0.05, * * P<0.01, * * * P<0.001$.

Two-tailed probability, $F$-test.

which includes the genetic variation component, was detected for all traits under poor nutrition and for wing length and bristle number under standard nutrition. For thorax length and wing length under poor nutrition, among-vial variation, which reflects the effect of the common environment, was highly significant. In thorax length the genetic (among lines) and environmental (among and within vials) variance components were higher under poor nutrition. The genetic variance of wing length did not differ between nutritional regimes, and both environmental variance components increased under nutritional stress. In bristle number, the variance components did not significantly differ between environments.

Table 4 gives the coefficients of intraclass correlation, which are used as measures of heritability $\left(h^{2}\right)$ in isofemale lines and sometimes are called isofemale heritability' (Parsons, 1983; intraclass correlation =
$1 /\left(2 / h^{2}+0.5\right)$. In all cases except thorax length under standard nutrition, they were statistically significant even after correction for multiple comparisons with the sequential Bonferroni method (Rice, 1989). Comparison between nutritional regimes revealed only one significant difference: the intraclass correlation in wing length was lower under poor than under standard nutrition.

\section{Genetic variation in mixed population}

Table 4 also shows offspring-mid-parent productmoment correlations for the three morphological traits in the mixed population. To measure heritability in this experiment, we used correlation coefficients instead of the traditionally used regression coefficients because regression values can lead to misleading heritability estimates when the variances in the parent and progeny 
Table 4 Intraclass correlations in isofemale lines $( \pm$ SE) and mean offspring-mid-parent correlations in mixed population $( \pm \mathrm{SE})$ for three morphological traits of Drosophila melanogaster

\begin{tabular}{llccc}
\hline Parameter & \multicolumn{1}{c}{ Trait } & Standard nutrition & Poor nutrition & Comparison, $P$ \\
\hline Intraclass & Thorax length & $0.060 \pm 0.060$ & $0.233 \pm 0.088^{* *}$ & 0.100 \\
$\quad$ correlation & Wing length & $0.848 \pm 0.030^{* * *}$ & $0.587 \pm 0.073^{* * *}$ & $<0.001$ \\
& Bristle number & $0.202 \pm 0.056^{* * *}$ & $0.256 \pm 0.062^{* * *}$ & 0.495 \\
Mean offspring- & Thorax length & $-0.026 \pm 0.082$ & $-0.074 \pm 0.082$ & - \\
mid-parent & Wing length & $0.337 \pm 0.077^{* * *}$ & $0.034 \pm 0.082$ & 0.007 \\
correlation & Bristle number & $0.188 \pm 0.081^{*}$ & $0.213 \pm 0.080^{*}$ & 0.824 \\
\hline
\end{tabular}

$* P<0.05, * * P<0.01,{ }^{* * *} P<0.001$, significance level after correcting for number of traits.

Two-tailed probability, $Z$-test.

generation differ (Ward, 1994). As noted above, the thorax length variances were different between generations in females under standard nutrition. In males, we also found significantly different (two-tailed $F$-tests, $P<0.01)$ thorax length and wing length variances both in the unstressed (parent variance $>$ offspring variance) and stressed (parent variance $<$ offspring variance) regimes. We initially correlated female and male parents separately, but because the variances did not differ between sexes and there were no maternal effects, we only present the mean offspring-mid-parent correlations. These correlations were not different from zero for thorax length in both nutritional regimes and for wing length under poor nutrition. Three other correlations, wing length under poor nutrition and bristle number in both nutritional regimes, were significantly different from zero even after the Bonferroni correction. As with the intraclass correlation, the mean offspring-midparent correlation was significantly higher for wing length under standard nutrition, whereas for bristle number, the differences between nutritional regimes were nonsignificant. Bearing in mind that heritability in isofemale lines would be at least twice the intraclass correlation (Parsons, 1983), one can see that in the mixed population estimates of this parameter are generally lower than in the isofemale lines (Table 4).

Estimates of genetic and environmental variances in wing length and bristle number in the mixed population exhibited the same trends as in the isofemale lines. However, their comparisons between nutritional regimes using the bootstrap technique detected no statistically significant differences. The genetic variances of wing length under standard vs. poor regimes were 3.97 vs. 0.79 (bootstrap-comparison, two-tailed $P=0.290$ ), the environmental variances, 8.47 vs. $14.65(P=0.130)$, respectively. For bristle number, the respective variance values were 0.26 vs. $0.24(P=0.920)$, and 0.96 vs. 0.98 $(P=0.910)$. As in the case of heritability, estimates of genetic variances in the mixed population were generally lower then in the isofemale lines (see Table 3).

\section{Discussion}

In our experiment, the direct stress indicators clearly showed that the limited-food conditions were stressful for the isofemale lines and for both generations of the mixed population: in all of them, viability and body size were highly significantly lower under poor than under standard nutrition. The behaviour of phenotypic variance was less conclusive. Although in some cases the variance was higher under poor nutrition, in other cases, it was not. It is possible that the stress level in the present work was not sufficient to produce significant changes in phenotypic variation. In another work dealing with nutritional stress in isofemale lines (Imasheva et al., 1999), viability in the low-food conditions was about $40 \%$ and clear differences in the phenotypic variance of thorax and wing length between stressful and control environments were recorded.

The estimates of genetic variation were in general higher in isofemale lines than in the offspring-parent design in either nutritional regime. Several non-alternative reasons can be suggested for explanation. First, increased heritabilities and genetic variances in isofemale lines may be associated with a more substantial contribution of nonadditive components obtained by this method as compared to the offspring-parent regression. The isofemale line analysis is a modification of the full-sib design; the full-sib covariance includes, in addition to the additive variance, nonadditive variance components due to dominance and epistasis (Falconer \& Mackay, 1996). The offspring-parents covariance does not include the dominance components and includes a far lesser proportion of the epistatic variance. Thus, if the dominant and epistatic components constitute a large part of the genetic variance, estimates of genetic variation in isofemale lines should be greater than in the offspring-parent comparisons.

Another possible explanation is an increase in amongline variation due to inbreeding. Hoffmann \& Parsons (1988), who proposed the isofemale line method for 
assessing heritability, predicted meaningful heritability estimates in the case when isofemale lines are maintained at an effective population size greater than 50 and tested within five generations after establishment. We estimated heritability in the fourth generation but maintained the lines using as parents 60 flies (30 females and 30 males), which in some lines might lead to an effective population size less than 50 .

In addition, lower heritability estimates in the offspring-parent design, obtained under stress, may be explained by fitness differences observed in our experiment. As evident from the viabilities, phenotypic variances and thorax length values, fitness in isofemale lines was lower than in the mixed population, which was probably caused by inbreeding within lines characteristic of the former design (Hoffmann \& Parsons, 1988). However, statistically significant differences in viability and body size were also observed between generations in the mixed population reared under poor nutrition. These differences could be a result of decreased fitness in offspring produced by stressed parents. Thus, we cannot exclude the situation that the three groups of flies (the isofemale lines, parental and offspring generations of the mixed population) exhibited different susceptibility to the stressor and consequently may be regarded as having experienced stresses of different intensity, which is equivalent to different environments. Regressing offspring values on the mid-parental ones yields unbiased heritability estimates only when both parents and offspring are reared in the same environment. If environments differ between the parental and the offspring generations, genotype-environment interaction may lead to lower heritability estimates (see Lande in Coyne \& Beecham, 1987; Riska et al., 1989).

The behaviour of genetic variation in the three morphological traits followed different scenarios but for each trait in both experimental designs it changed under stress in a similar way. The most clear-cut results were obtained for sternopleurals. In this trait, neither phenotypic nor genetic variances exhibited stressinduced changes; consequently, heritabilities did not change either. The same results for this trait were obtained earlier for nutritional stress by the isofemale line method (Imasheva et al., 1999), and using offspring-parent comparisons and a combined stress consisting of poor nutrition (low yeast concentration), ethanol added to the medium, and repeated cold shock (Hoffmann \& Schiffer, 1998). However, Bubliy et al. (2000b), who previously analysed the population used here with the half-sib technique, found a decrease in genetic variance under poor nutrition, whereas the phenotypic variance remained constant.

Heritability of wing length in the present work and in the studies of Hoffmann \& Schiffer (1998) and Imasheva et al. (1999) was lower in the stressful environment because of a drastic increase in the environmental component of the phenotypic variation. Genetic variance of this trait did not differ between environments in our study and in the experiments of Hoffmann \& Schiffer (1998) and Bubliy et al. (2000b). By contrast, Imasheva et al. (1999) recorded that both genetic and environmental variance components were higher under food-limited conditions. De Moed et al. (1997), who used a low yeast content and low temperature as a combined stress, showed that the genetic variance under stress increased and the environmental variance did not change.

In thorax length, no significant differences in heritabilities between environments were found in our work and in the studies of Imasheva et al. (1999) and Bubliy et al. (2000b). Four of six heritability estimates in the population tested (including data from Bubliy et al., 2000 b) did not differ from zero, which does not seem an artifact in view of the relatively low level of heritability of this trait (Roff \& Mousseau, 1987). However, in the present study, as well as in the works of De Moed et al. (1997) and Imasheva et al. (1999), the genetic variance of thorax length in isofemale lines was increased under stress, and the differences between the normal and stressful environments were generally extremely pronounced (about an order of magnitude). A stressinduced increase in the environmental variance, if any, was less dramatic then in the genetic variance.

It can be seen that the pattern of genetic variation of thorax length is similar to that of life-history traits for which higher heritability under stress is often recorded (Hoffmann \& Parsons, 1991; Sgrò \& Hoffmann, 1998a,b). Moreover, the thorax length directly measures body size. The latter is positively correlated to several fitness components (for references, see Partridge \& Fowler, 1993) and may be the morphological trait in Drosophila most closely related to fitness. As a speculation, we may suggest that in both cases higher genetic variation under stress is associated with an increase in the epistatic rather than in the additive variance component. Both body size and life-history traits are controlled by numerous genes, which enhances the probability of between-gene interaction. The effect of stress on genetic variation in thorax length is stronger in isofemale lines, where the heritability estimates include a substantial fraction of dominance/epistatic variance. For life-history traits there is direct evidence indicating higher expression of nonadditive variation in Drosophila under stress (Blows \& Sokolowski, 1995). In any case, our results warrant caution in making generalizations about changes of genetic variance across environments based on data obtained for a particular trait. This includes conclusions on variation in body size some- 
times made on the basis of wing-length data. The differences between traits may reflect crucial features of their genetic architecture and biological significance.

\section{Acknowledgements}

We thank Doth Andersen for technical assistance and Gerdien de Jong, Stuart Barker, Jean David and two anonymous reviewers for critical comments on the manuscript. This work was supported by the Danish Natural Science Research Council (grant nos 9700177 and 9701412) and the NATO Collaboration Research Programme (Linkage Grant CRG.LG.972856).

\section{References}

BARKER, J. S. F. AND KREBS, R. A. 1995. Genetic variation and plasticity of thorax length and wing length in Drosophila aldrichi and D. buzzatii. J. Evol. Biol., 8, 689-709.

BLows, M. W. AND SOKOLOWSKI, M. B. 1995. The expression of additive and nonadditive genetic variation under stress. Genetics, 140, 1149-1159.

BLum, A. 1988. Plant Breeding for Stress Environments. CRC Press, Boca Raton.

BUbliy, O. A., LOESCHCKE, v. AND IMASHEVA, A. G. 2000a. Effect of stressful and nonstressful growth temperatures on variation of sternopleural bristle number in Drosophila melanogaster. Evolution, 54, 1444-1449.

BUbliy, O. A., IMASHEVA, A. G., LOESCHCKe, v. 2000b. Half-sib analysis of three morphological traits in Drosophila melanogaster under poor nutrition. Hereditas, 133, 59-63.

COYNE, J. A. AND BEECHAM, E. 1987. Heritability of two morphological characters within and among natural populations of Drosophila melanogaster. Genetics, 117, 727-737.

DE MOED, G. H., DE JONG, G. AND SCHARLOO, W. 1997. Environmental effects on body size variation in Drosophila melanogaster and its cellular basis. Genet. Res., 70, 35-43.

FALCONER, D. S. AND MACKAY, T. F. C. 1996. Introduction to Quantitative Genetics, 4th edn. Longman, Harlow.

FORBES, V. E. AND CALOW, P. 1997. Responses of aquatic organisms to pollutant stress: theoretical and practical implications. In: Bijlsma, R. and Loeschcke, V. (eds) Environmental Stress, Evolution and Adaptation, pp. 25-32. Birkhäuser Verlag, Basel.

HOFFMANN, A. A. AND MERILÄ, J. 1999. Heritable variation and evolution under favourable and unfavourable conditions. Trends Ecol. Evol., 14, 96-101.

HOFFMANN, A. A. AND PARSONS, P. A. 1988. The analysis of quantitative variation in natural populations with isofemale strains. Génét. Sél. Evol., 20, 87-98.
HOFFMANN, A. A. AND PARSONS, P. A. 1991. Evolutionary Genetics and Environmental Stress. Oxford University Press, Oxford.

HOLLOWAY, G. J., POVEY, S. R. AND SIBLY, R. M. 1990. The effect of new environment on adapted genetic architecture. Heredity, 64, 323-330.

IMASHEVA, A. G., LOESCHCKE, V., ZHIVOTOVSKY, L. A. AND LAZEBNY, O. E. 1998. Stress temperatures and quantitative variation in Drosophila melanogaster. Heredity, 81, 246-253.

IMASHEVA, A. G., BOSENKO, D. V. AND BUBLI, O. A. 1999. Variation in morphological traits of Drosophila melanogaster (fruit fly) under nutritional stress. Heredity, 82, 187-192.

LOESCHCKE, V., BUNDGAARD, J. AND BARKER, J. F. S. 1999. Reaction norms across and genetic parameters at different temperatures for thorax and wing size traits in Drosophila aldrichi and D. buzzatii. J. Evol. Biol., 12, 605-623.

MATHER, K. 1943. Polygenic inheritance and natural selection. Biol. Rev., 18, 32-64.

Møller, A. P. AND SwADDle, J. P. 1997. Asymmetry, Developmental Stability and Evolution. Oxford University Press, Oxford

PARSONS, P. A. 1983. The Evolutionary Biology of Colonizing Species. Cambridge University Press, New York.

PARTRIDGE, L. AND FOWLER, K. 1993. Direct and correlated responses to selection on thorax length in Drosophila melanogaster. Evolution, 47, 213-226.

PIGLIUCCI, M., SCHLichting, C. D. AND WhitTON, J. 1995. Reaction norms of Arabidopsis. II. Response to stress and unordered environmental variation. Funct. Ecol., 9, 537-547.

RICE, W. R. 1989. Analyzing tables of statistical tests. Evolution, 43, 223-225.

RISKA, B., PROUT, T. AND TURELLI, M. 1989. Laboratory estimates of heritabilities and genetic correlation in nature. Genetics, 123, 865-871.

ROFF, D. A. AND MOUSSEAU, T. A. 1987. Quantitative genetics and fitness: lessons from Drosophila. Heredity, 58, 103-118.

SGRÒ, C. M. AND HOFFMANN, A. A. 1998a. Effects of temperature extremes on genetic variances for life history traits in Drosophila melanogaster as determined from parent-offspring comparisons. J. Evol. Biol., 11, 1-20.

SGRÒ, C. M. AND HOFFMANN, A. A. 1998b. Effects of stress combinations on the expression of additive genetic variation for fecundity in Drosophila melanogaster. Genet. Res., 72 , $13-18$.

WARD, P. J. 1994. Parent-offspring regression and extreme environments. Heredity, 74, 80-90.

WOODS, R. E., SGRÒ, C. M., HERCUS, M. J. AND HOFFMANN, A. A. 1999. The association between fluctuating asymmetry, trait variability, trait heritability, and stress: a multiply replicated experiment on combined stresses in Drosophila melanogaster. Evolution, 53, 493-505.

ZAR, J. H. 1996. Biostatistical Analysis, 3rd ed. Prentice Hall, Upper Saddle River. 